\title{
Effect of Implementing Nursing Guideline on Emergency Hypertensive Patients' Outcomes at Healthy Insurance Hospital in Assuit.
}

\author{
Hayam Abd El-Monam Ahmed, Hanan Mohmoud Ahmed, Mona Aly Mohammed \& Asmaa Aly Mahgoub. \\ Head Nurse in coronary Care unit, Healthy Insurance hospital, Assuit, Egypt. \\ Professor of Internal Medicine, Faculty of Medicine, Assuit University, Egypt. \\ Lecturer of Critical Nursing, Faculty of Nursing, Assuit University, Egypt. \\ Lecturer of Critical Nursing, Faculty of Nursing, Assuit University, Egypt.
}

\begin{abstract}
Background: Emergency hypertension is elevation of blood pressure (Systolic over 180 or diastolic over 120) associated with acute end organ damage .The critical care nurse assumes important role for the care and clinical stabilization of the patient with emergency hypertension Aim this study was carried out to evaluate the effect of nursing guideline on patient's outcomes with hypertension emergency. Setting This study was conducted at coronary care unit. Subjects A convenient sample of (60) patients that divided in to two groups (control 30 and study 30) collected over threeteen months. Tools Two tools were utilized to collect data pertinent to study Tool I Socio demographic and clinical data assessment sheet. Tool II Sequential Organ Failure Assessment scale "SOFA" score. Nursing guideline for patient with emergency hypertension Methods emergency hypertensive nursing guideline implemented by researcher using Tools I, II from the first day of admission and until discharge then the data were recorded in the developed tools. Results As regards to the emergency hypertensive patients suffering neurological manifestations, results revealed that there were statistically significant difference between studied groups in first day $(\mathrm{P}=0.046)$. As regards to the emergency hypertensive patients suffering renal manifestations, results revealed that there were statistically significant difference between studied groups in third and fourth day $(\mathrm{P}=0.048$ and $\mathrm{P}=0.019)$ respectively. Conclusion Applying nursing care guideline for patients with emergency hypertension showed statistically significance difference in reduction incidence of complication regarding kidney function in third and fourth day and length of hospital stay.
\end{abstract}

\section{Keywords: Emergency Hypertension, Nursing Guideline \& Patient Outcomes.}

\section{Introduction}

A hypertensive emergency (Systolic over 180 or diastolic over 120)(formerly called "malignant hypertension") is hypertension with acute impairment of one or more organ systems (especially the central nervous system, cardiovascular system and/or the renal system) that can result in irreversible organ damage. In a hypertensive emergency, the blood pressure should be slowly lowered over a period of minutes to hours with an antihypertensive agent (Sinha, 2011).

Recent data suggest that systemic hypertension is the responsible cause for nearly 7.1 million deaths per year worldwide. Approximately 1-2\% of patients with hypertension will develop a hypertensive crisis which can be further categorized as either hypertensive emergency or urgency depending on either the presence or absence of acute end-organ dysfunction, respectively. Hypertensive crises can develop in patients with or without preexisting chronic hypertension (Mohan \& Nelson-Piercy, 2016).

Hypertensive emergency is characterized by a marked rapid increase in blood pressure that initially leads to intense vasoconstriction as the body attempts to protect itself from the elevated blood pressure. If the blood pressure remains critically high ,compensatory vasoconstriction fails, resulting in increased pressure and blood flow throughout the vascular system . Hypertensive emergency is associated with a variety of clinical situations as acute cerebrovascular syndrome, aortic dissection, myocardial infarction and kidney failure (Mancia \& Fagard, 2014).

Hypertensive emergency can manifest as any the following symptoms depending on the target organ involved: central nervous system comprise, identified by headache, blurred vision, change in level of consciousness, or coma .Cardiovascular comprise, identified by chest pain of acute coronary syndrome or aortic dissection. Acute kidney failure, identified by sudden absence of urine out put(Gupta et al., 2009).

The focus of nursing management for the patient with hypertensive emergency is to return the blood pressure to the desired range and improve patient outcomes. After the hypertension is controlled, the nurse identifies the factors that resulted in this lifethreatening condition. Several nursing diagnoses are associated with hypertensive crisis (Lewis et al., 2016). 
Critical care nurse should assess the patient age, severity and duration of hypertension, physical examination and laboratory findings, prior treatment of the hypertension ( drug type , dose , side effect ), intake of agent that may cause hypertension ( oral contraceptive , sympathomimetic ) family history , symptoms of target organ damage( headache, loss of vision acuity, chest pain ,dyspnea , or claudication ), presence of risk Factor, dietary history and psychosocial factors (Carlson.,2011).

Nursing guideline can change the process of nurse's decision making .Clinical guideline can change the process of health care and improve patient's out comes. Well-constructed and up to date clinical guideline provide the nurse with a framework for offering the highest standards of nursing care against which nurses can monitor their own clinical practice (Stevens ,2013).

\section{Significance of the study}

According to the reports of Health Insurance hospital in Assuit the number of hypertensive patients admitted in(2012-2013) was 89,In (2013-2014) it was 97, and in (2014-2015) it was 114patients 85 from these patients diagnosed with emergency hypertension(Healthy Insurance hospital reports,2015).

Among the adult population of Egypt aged 25 to 59, the prevalence of hypertension was $57.2 \%(0.6)$ and $17.6 \%(0.5)$ respectively. Only $25.2 \%$ of the population had normal blood pressure levels of $<120 / 80$ mmHg (Hasan, et al., 2014).

Across the WHO regions, the prevalence of raised blood pressure was highest in Africa, where it was $46 \%$ for both sexes combined. Both men and women have high rates of raised blood pressure in the Africa region, with prevalence rates over $40 \%$. The lowest prevalence of raised blood pressure was in the WHO Region of the Americas at $35 \%$ for both sexes(WHO,2013).

\section{Aim of the Study}

To evaluate the effect of nursing guidelines on patient's outcomes with hypertensive emergency at Healthy Insurance hospital in Assuit.

\section{Research hypotheses}

- Length of stay of patients who exposed to nursing guideline care will be less than patients who didn't exposed to nursing guideline care.

- A significant reduction in the incidence of complications occurrence will be in study group compared to control group of patients.

\section{Subjects \& methods \\ Research design}

The present study used quasi-experimental research design.

\section{Setting of the study}

The study was conducted at the coronary care unit of Healthy Insurance hospital in Assuit.

\section{Sampling}

Data were collected over threeteen months(from December 2015 to December 2016)and the sample was convenient sample of patients (60 patients) who were admitted at coronary care unit (15 beds) and diagnosed with emergency hypertension, divided into (30 patients) study group and (30 patients) control group.

\section{Tools of the study}

Three tools were used to collect the data in this study and developed by researcher based on review of literature.

Tool (I) "Socio-demographic and clinical data assessment sheet "This tool consist of: Sociodemographic data about the patient such as (code number, age, sex, marital status, diagnosis, date of admission. to asses patient profile and clinical data assessment sheet to assess the clinical state of patients with emergency hypertension: (presence of a history of hypertension, family history, previous antihypertensive medications and its doses, recent symptoms, the initial measurement of blood pressure in admission and medication given to patient).

Tool (II) "Sequential Organ Failure Assessment"(SOFA) Scales.The Sequential Organ Failure Assessment (SOFA) scale is a mortality prediction scale that is based on the degree of dysfunction of 6 organ systems. Modified by (Vincent,2015). The scale is calculated on admission and every 24 hours until discharge using the worst parameters measured during the prior 24 hours. The scale can be used in a number of ways: As individual scales for each organ to determine the progression of organ dysfunction. As the sum of scales on one single ICU day. As the sum of the worst scales during the ICU stay. It is believed to provide a better stratification of the mortality risk in ICU patients given that the data used to calculate the scale is not restricted to admission values. The SOFA scale can be used to determine the level of organ dysfunction and the mortality risk in ICU patients. SOFA scale can be used to determine the level of organ dysfunction of the following organs : respiratory system, nervous system, cardiovascular system , liver, coagulation, and kidney function .

"Nursing guidelines for patient with emergency hypertension"This tool was prepared by the researcher after reviewing literature and articles including nursing guidelines regarding emergent hypertension (standardized assessment for patient clinical data and medical history, standardized blood pressure measuring technique, attaching the patient to the monitor to facilitate following patient vital signs 
and IV line should be inserted for giving medication, a blood sample was obtained to investigate renal function, hepatic function, $\mathrm{CBC}$, lipogram and biocardiac marker on admission, ECG, CT scan was performed, assessment degree of end-organ damage using SOFA scale, fluid intake and output chart should be assessed every hour, reassess BP after 5 minutes of admission , after 10 minutes, after 15 minutes, after 20 minutes, after 25 minutes, after 30 minutes, after 45 minutes, after 60 minutes then measuring BP every hour after stabilization of the case and applying nursing care specific to each diagnosis as the following : for cardiac patients who suffer from chest pain related to cardiac tissue ischemia ,For patients with hypertensive emergency who suffer from renal disorders (risk for ineffective renal perfusion), For patients who suffer from stroke (risk for ineffective cerebral tissue perfusion), For all patients with a hypertensive emergency (anxiety related to unpredictable nature of condition and fear of death), ineffective management related to lack of knowledge of condition, diet restrictions, medications, risk factors, and follow-up care) and patient education for all patients with hypertensive emergency. to compare effect of nursing guidelines regarding emergent hypertension on patients outcomes verses routine hospital nursing care regarding emergent hypertension.

Methods

- An official permission was obtained from Dean of the Faculty of Nursing Assuit University and Administrative manager of Healthy Insurance hospital in Assuit.

- Ethical consideration the nature and purpose of the study were explained to every patient.

- Consent was taken from participating personnel, after explaining the nature and purpose of the study.

- Confidentiality and anonymity of the subjects assured through coding the data.

- The tools (I) used in the study was developed by the researcher based on reviewing the relevant literature.

- Content validity: The developed tools (I) was tested for content validity by a jury of (5) specialists in the field of Internal Medicine and critical care nursing from Assuit University, and necessary modifications were done.

- The pilot study: was carried out in order to assess the feasibility and applicability of the tools and necessary modifications were done. The pilot study was done on $10 \%$ of patients who were included in the study.
- The studied sample fulfilling research criteria was assigned to two groups (the control group and the study group).

- The control group received usual care of hospital without Intervention from researcher.

- The study group received hypertensive emergency nursing care guidelines.

Research implementation(Procedure)

This study was carried out in three phases

Phase I: Preparatory Phase

A review of current and past, local and international related literature in the various aspect using books, articles , and periodicals, the journals was done .The proposed study setting was assessed for a number of patients admitted to coronary care unit suffering from hypertension emergency, this ended by a pilot study.

\section{Phase II: implementation phase}

Data were collected In Coronary Care unit at Healthy Insurance hospital during December 2015 -December 2016, The data was collected from the first day of admission and until discharge then the data were recorded in the developed tools.

\section{For the control group}

Patients who were received routine hospital care for emergency hypertensive patients from first day of admission until discharge.

\section{For study group}

- The researcher assessed patients then applying emergency hypertension nursing care guideline.

- The nursing care guideline implementation by researcher using Tools I, II from the first day of admission and until discharge then the data were recorded in the developed tools.

- The researcher measured BP using standardized technique.

- The researcher attached patient to the monitor, blood sample was obtained for investigations.

- The researcher assessed degree of end-organ damage using "SOFA scale" assessment of cardiovascular system, renal system and neuromuscular system. Then applying nursing care specific to the affected organ.

\section{Evaluation Phase}

This phase was done to evaluate effect of applying emergency hypertension nursing guidelines on patients' outcomes by comparing (Length of stay and presence of complications)between study and control groups using tool (II). 


\section{Results}

Table1: Percent distribution of demographic data and medical history of control and study groups.

\begin{tabular}{|c|c|c|c|c|c|}
\hline \multirow{2}{*}{ demographic data } & \multicolumn{2}{|c|}{$\overline{\text { Control }}$} & \multicolumn{2}{|c|}{ Study } & \multirow{2}{*}{ P. value } \\
\hline & No. $=(30)$ & $\%$ & No. $=(30)$ & $\%$ & \\
\hline Age & \multicolumn{2}{|c|}{$58.8 \pm 4.5$} & \multicolumn{2}{|c|}{$58.7 \pm 5.6$} & $0.980^{\mathrm{ns}}$ \\
\hline \multicolumn{6}{|l|}{ Diagnosis } \\
\hline Heart failure & 4 & 13.3 & 2 & 6.7 & \multirow{7}{*}{$0.774^{\mathrm{ns}}$} \\
\hline CVA & 3 & 10 & 1 & 3.3 & \\
\hline Hypertensive encephalopathy & 2 & 6.7 & 2 & 6.7 & \\
\hline Myocardial infraction & 3 & 10.0 & 3 & 10.0 & \\
\hline Pulmonary edema & 6 & 20.0 & 9 & 30.0 & \\
\hline Renal impairment & 6 & 20.0 & 4 & 13.3 & \\
\hline Unstable Angina & 6 & 20.0 & 9 & 30.0 & \\
\hline \multicolumn{6}{|l|}{ Medical history } \\
\hline $\mathrm{DM}$ & 8 & 26.7 & 13 & 43.3 & $0.207^{\mathrm{ns}}$ \\
\hline Cardiac disease & 9 & 30 & 7 & 23.3 & $0.819^{\mathrm{ns}}$ \\
\hline Respiratory disease & 13 & 43.3 & 11 & 36.6 & $0.712^{\mathrm{ns}}$ \\
\hline
\end{tabular}

* Statistically significant difference $(p<0.05)$

CVA: Cerebral Vascular Accident

Ns: Non significant

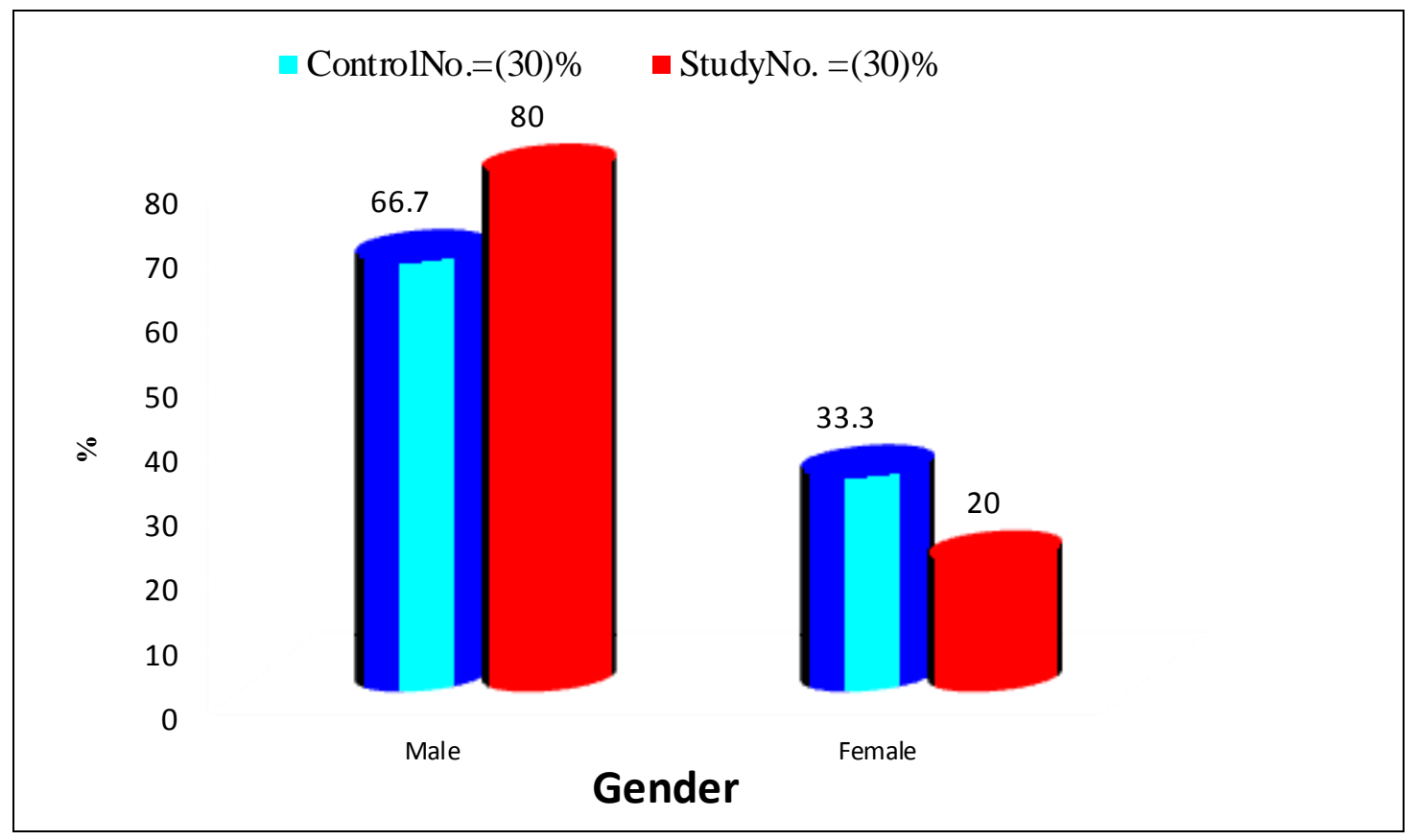

Figure (1): Distribution of control and study groups related to gender. 


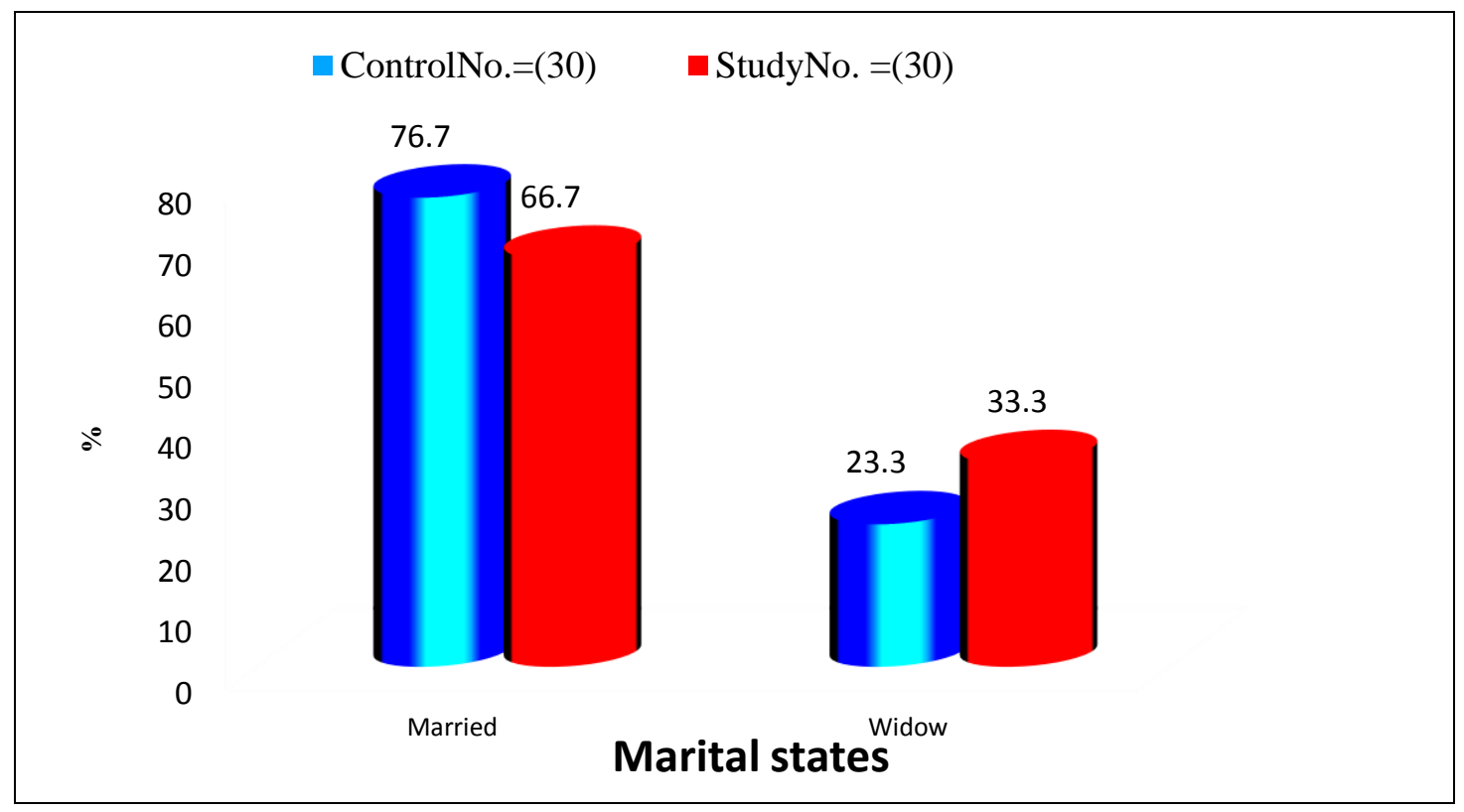

Figure ( 2): Distribution of control and study groups related to marital states.

Table (2): Distribution of control and study groups regards respiratory and cardiovascular assessment.

\begin{tabular}{|c|c|c|c|c|c|}
\hline \multirow{2}{*}{$\begin{array}{c}\text { Respiratory and cardiovascular } \\
\text { assessment }\end{array}$} & \multicolumn{2}{|c|}{ Control } & \multicolumn{2}{|c|}{ Study } & \multirow{2}{*}{ P. value } \\
\hline & $\mathrm{No}=(30)$ & $\%$ & $\mathrm{No}=(30)$ & $\%$ & \\
\hline HR & \multicolumn{2}{|c|}{$98.7 \pm 16.1$} & \multicolumn{2}{|c|}{$104.5 \pm 13.7$} & $0.130^{\mathrm{ns}}$ \\
\hline \multicolumn{6}{|l|}{ Rhythm } \\
\hline Regular & 30 & 100.0 & 30 & 100.0 & \multirow{2}{*}{ - } \\
\hline Irregular & 0 & 0.0 & 0 & 0.0 & \\
\hline \multicolumn{6}{|c|}{ On admission Blood pressure measurement } \\
\hline \multicolumn{6}{|l|}{ SBP } \\
\hline Right arm & \multicolumn{2}{|c|}{$219.5 \pm 17.34$} & \multicolumn{2}{|c|}{$227.63 \pm 17.19$} & $0.158^{\mathrm{ns}}$ \\
\hline Left arm & \multicolumn{2}{|c|}{$215.68 \pm 16.56$} & \multicolumn{2}{|c|}{$224.00 \pm 24.99$} & $0.139^{\mathrm{ns}}$ \\
\hline Standing BP & \multicolumn{2}{|c|}{$216.7 \pm 16.9$} & \multicolumn{2}{|c|}{$210 \pm 17.6$} & $0.064^{\mathrm{ns}}$ \\
\hline \multicolumn{6}{|l|}{ DBP } \\
\hline Right arm & \multicolumn{2}{|c|}{$227.6 \pm 17.19$} & \multicolumn{2}{|c|}{$219.95 \pm 12.13$} & 0.151 \\
\hline Left arm & \multicolumn{2}{|c|}{$113.24 \pm 10.09$} & \multicolumn{2}{|c|}{$119.00 \pm 13.13$} & 0.0161 \\
\hline Standing BP & \multicolumn{2}{|c|}{$113.9 \pm 11.7$} & \multicolumn{2}{|c|}{$119.5 \pm 12.1$} & 0.173 \\
\hline Respiratory Rate & \multicolumn{2}{|c|}{$22.3 \pm 4.1$} & \multicolumn{2}{|c|}{$23.9 \pm 5.2$} & $0.191^{\mathrm{ns}}$ \\
\hline \multicolumn{6}{|l|}{ Dysrhythmia } \\
\hline Present & 0 & 0 & 0 & $\mathbf{0}$ & \multirow[b]{2}{*}{-} \\
\hline Absent & 30 & 100.0 & 30 & 100.0 & \\
\hline \multicolumn{6}{|l|}{ Cyanosis } \\
\hline Present & 0 & 0 & 0 & 0 & \multirow[b]{2}{*}{-} \\
\hline Absent & 30 & 100.0 & 30 & 100.0 & \\
\hline \multicolumn{6}{|l|}{ Chest } \\
\hline Normal & 9 & 30.0 & 8 & 26.6 & \multirow{3}{*}{$0.317^{\mathrm{ns}}$} \\
\hline Basal crepetations & 5 & 16.7 & 10 & 33.3 & \\
\hline other specify(chest pain) & 16 & 53.3 & 12 & 40.0 & \\
\hline
\end{tabular}

* Statistically significant difference $(p<0.05)$

HR: Heart Rate

BP: Blood Pressure

SBP: Systolic Blood Pressure

DBP: Diastolic Blood Pressure 
Table (3) Distribution of control and study groups regards Surgical and family history.

\begin{tabular}{|c|c|c|c|c|c|}
\hline \multirow{2}{*}{ Surgical and family history } & \multicolumn{2}{|c|}{ control } & \multicolumn{2}{|c|}{ Study } & \multirow{2}{*}{ P. value } \\
\hline & $\mathrm{No}=(30)$ & $\%$ & $\mathrm{No}=(30)$ & $\%$ & \\
\hline \multicolumn{6}{|l|}{ Surgical history } \\
\hline Yes & 8 & 26.7 & 5 & 16.7 & \multirow{2}{*}{$0.347^{\mathrm{ns}}$} \\
\hline No & 22 & 73.3 & 25 & 83.3 & \\
\hline \multicolumn{6}{|l|}{ Family history } \\
\hline HTN & 19 & 30.0 & 16 & 53.3 & $0.189^{\mathrm{ns}}$ \\
\hline Diabetes Mellitus & 17 & 56.7 & 18 & 60.0 & $0.793^{\mathrm{ns}}$ \\
\hline Sudden death & 4 & 13.3 & 3 & 10.0 & $0.688^{\mathrm{ns}}$ \\
\hline CAD & 0 & 0.0 & 0 & 0.0 & - \\
\hline
\end{tabular}

* Statistically significant difference ( $p<0.05)$

DM: Diabetes Mellitus HTN: Hypertension CAD: Coronary Artery Disease

Table( 4): Distribution of control and study groups regards temperature, heart rate and respiratory rate assessment.

\begin{tabular}{|c|c|c|c|c|c|}
\hline \multirow{2}{*}{$\begin{array}{l}\text { Temperature, Heart rate and } \\
\text { Respiratory rate assessment. }\end{array}$} & \multicolumn{2}{|c|}{ Control } & \multicolumn{2}{|c|}{ Study } & \multirow{2}{*}{ P. value } \\
\hline & $\mathrm{No}=(30)$ & $\%$ & $\mathrm{No}=(30)$ & $\%$ & \\
\hline \multicolumn{6}{|l|}{ Temperature } \\
\hline $1^{\text {st }}$ day & \multicolumn{2}{|c|}{$37.1 \pm 0.4$} & \multicolumn{2}{|c|}{$37.2 \pm 0.5$} & $0.882^{\text {ns }}$ \\
\hline $2^{\text {nd }}$ day & \multicolumn{2}{|c|}{$37.1 \pm 0.5$} & \multicolumn{2}{|c|}{$37.2 \pm 0.3$} & $0.791^{\mathrm{ns}}$ \\
\hline $3^{\text {rd }}$ day & \multicolumn{2}{|c|}{$37.0 \pm 0.3$} & \multicolumn{2}{|c|}{$37.1 \pm 0.4$} & $0.457^{\mathrm{ns}}$ \\
\hline $4^{\text {th }}$ day & \multicolumn{2}{|c|}{$37.0 \pm 0.2$} & \multicolumn{2}{|c|}{$37.1 \pm 0.2$} & $0.419^{\mathrm{ns}}$ \\
\hline \multicolumn{6}{|l|}{ HR } \\
\hline $1^{\text {st }}$ day & \multicolumn{2}{|c|}{$99.7 \pm 13.1$} & \multicolumn{2}{|c|}{$102.2 \pm 13.7$} & $0.221^{\mathrm{ns}}$ \\
\hline $2^{\text {nd }}$ day & \multicolumn{2}{|c|}{$99.9 \pm 12.0$} & \multicolumn{2}{|c|}{$102.9 \pm 14.1$} & $0.312^{\mathrm{ns}}$ \\
\hline $3^{\text {rd }}$ day & \multicolumn{2}{|c|}{$100.1 \pm 12.0$} & \multicolumn{2}{|c|}{$101.3 \pm 14.1$} & $0.721^{\mathrm{ns}}$ \\
\hline $4^{\text {th }}$ day & \multicolumn{2}{|c|}{$99.5 \pm 12.0$} & \multicolumn{2}{|c|}{$99.9 \pm 14.1$} & $0.881^{\mathrm{ns}}$ \\
\hline \multicolumn{6}{|l|}{ Respiratory Rate } \\
\hline $1^{\text {st }}$ day & \multicolumn{2}{|c|}{$22.1 \pm 3.7$} & \multicolumn{2}{|c|}{$23.3 \pm 5.1$} & $0.194^{\mathrm{ns}}$ \\
\hline $2^{\text {nd }}$ day & \multicolumn{2}{|c|}{$22.4 \pm 3.1$} & \multicolumn{2}{|c|}{$23.1 \pm 5.1$} & $0.237^{\mathrm{ns}}$ \\
\hline $3^{\text {rd }}$ day & \multicolumn{2}{|c|}{$22.3 \pm 3.5$} & \multicolumn{2}{|c|}{$22.9 \pm 4.3$} & $0.411^{\mathrm{ns}}$ \\
\hline $4^{\text {th }}$ day & \multicolumn{2}{|c|}{$22.2 \pm 3.3$} & \multicolumn{2}{|c|}{$23.0 \pm 4.8$} & $0.319^{\mathrm{ns}}$ \\
\hline
\end{tabular}

* Statistically significant difference $(p<0.05)$

Table (5 ): Distribution of control and study groups regards blood pressure assessment after nursing guidelines implementation.

\begin{tabular}{|c|c|c|c|c|c|c|}
\hline \multirow{2}{*}{$\begin{array}{l}\text { Blood Pressure } \\
\text { assessment. }\end{array}$} & \multicolumn{2}{|c|}{ SBP } & \multirow[b]{2}{*}{ P. value } & \multicolumn{2}{|c|}{ DBP } & \multirow[b]{2}{*}{ P. value } \\
\hline & $\begin{array}{l}\text { Control } \\
\mathrm{N}=(\mathbf{3 0})\end{array}$ & $\begin{array}{l}\text { Study } \\
\mathrm{N}=(\mathbf{3 0})\end{array}$ & & $\begin{array}{l}\text { Control } \\
\mathrm{N}=(\mathbf{3 0})\end{array}$ & $\begin{array}{c}\text { Study } \\
\mathrm{N}=(\mathbf{3 0})\end{array}$ & \\
\hline Baseline & $215.5 \pm 17.34$ & $227.63 \pm 17.19$ & $0.009 * *$ & $127.6 \pm 17.19$ & $121.00 \pm 12.13$ & $0.028 *$ \\
\hline $5 \min$ & $242.24 \pm 2.52$ & $237.33 \pm 3.40$ & $0.209^{\mathrm{ns}}$ & $122.23 \pm 3.11$ & $127.32 \pm 2.01$ & $0.086^{\mathrm{ns}}$ \\
\hline $10 \mathrm{~min}$ & $237.37 \pm 2.42$ & $232.32 \pm 2.29$ & $0.359^{\mathrm{ns}}$ & $124.41 \pm 4.18$ & $127.33 \pm 2.13$ & $0.114^{\mathrm{ns}}$ \\
\hline $15 \min$ & $227.29 \pm 2.33$ & $225.33 \pm 2.23$ & $0.473^{\mathrm{ns}}$ & $117.28 \pm 2.12$ & $122.35 \pm 5.24$ & $0.086^{\mathrm{ns}}$ \\
\hline $20 \mathrm{~min}$ & $222.18 \pm 2.18$ & $219.31 \pm 4.27$ & $0.228^{\mathrm{ns}}$ & $114.31 \pm 2.25$ & $117.43 \pm 2.19$ & $0.428^{\mathrm{ns}}$ \\
\hline $25 \min$ & $217.32 \pm 3.23$ & $212.33 \pm 3.92$ & $0.107^{\mathrm{ns}}$ & $97.43 \pm 2.31$ & $102.37 \pm 2.08$ & $0.310^{\mathrm{ns}}$ \\
\hline $30 \mathrm{~min}$ & $201.61 \pm 2.14$ & $197.34 \pm 1.37$ & $0.219^{\mathrm{ns}}$ & $97.29 \pm 3.39$ & $102.33 \pm 4.13$ & $0.109^{\mathrm{ns}}$ \\
\hline
\end{tabular}




\begin{tabular}{|c|c|c|c|c|c|c|}
\hline \multirow{2}{*}{$\begin{array}{c}\text { Blood Pressure } \\
\text { assessment. }\end{array}$} & \multicolumn{2}{|c|}{ SBP } & \multirow[b]{2}{*}{ P. value } & \multicolumn{2}{|c|}{ DBP } & \multirow[b]{2}{*}{ P. value } \\
\hline & $\begin{array}{l}\text { Control } \\
\mathrm{N}=(\mathbf{3 0})\end{array}$ & $\begin{array}{c}\text { Study } \\
\mathrm{N}=(\mathbf{3 0})\end{array}$ & & $\begin{array}{l}\text { Control } \\
\mathrm{N}=(\mathbf{3 0})\end{array}$ & $\begin{array}{c}\text { Study } \\
\mathrm{N}=(\mathbf{3 0})\end{array}$ & \\
\hline $45 \min$ & $197.54 \pm 2.37$ & $177.41 \pm 3.49$ & $0.001 * *$ & $92.34 \pm 2.22$ & $84.31 \pm 2.48$ & $0.005^{* *}$ \\
\hline $60 \mathrm{~min}$ & $177.23 \pm 4.51$ & $167.53 \pm 2.34$ & $0.007 * *$ & $92.51 \pm 4.14$ & $83.75 \pm 3.39$ & $0.003 * *$ \\
\hline $1^{\text {st }}$ day & $167.68 \pm 3.22$ & $147.33 \pm 2.25$ & $0.001 * *$ & $87.41 \pm 2.31$ & $77.41 \pm 2.02$ & $0.007 * *$ \\
\hline $2^{\text {nd }}$ day & $157.67 \pm 2.17$ & $141.37 \pm 2.28$ & $0.331^{\mathrm{ns}}$ & $87.72 \pm 2.65$ & $83.33 \pm 2.37$ & $0.101^{\mathrm{ns}}$ \\
\hline $3^{\text {rd }}$ day & $141.43 \pm 2.25$ & $137.30 \pm 4.30$ & $0.081^{\mathrm{ns}}$ & $82.46 \pm 4.01$ & $77.69 \pm 2.92$ & $0.060^{\mathrm{ns}}$ \\
\hline Day of discharge & $119.87 \pm 2.23$ & $117.42 \pm 3.27$ & $0.213^{\mathrm{ns}}$ & $82.13 \pm 2.71$ & $77.23 \pm 3.11$ & $0.060^{\mathrm{ns}}$ \\
\hline
\end{tabular}

* Statistically significant difference $(p<0.05)$

Table (6): Percentage distribution of control and study groups regards SOFA score according to Nervous system after nursing guidelines implementation.

\begin{tabular}{|c|c|c|c|c|c|}
\hline \multirow{2}{*}{ Glasgow coma scale } & \multicolumn{2}{|c|}{ Control } & \multicolumn{2}{|c|}{ Study } & \multirow[t]{2}{*}{ P. Value } \\
\hline & $\mathrm{No}=(30)$ & $\%$ & $\mathrm{No}=(30)$ & $\%$ & \\
\hline \multicolumn{6}{|l|}{$\mathbf{1}^{\text {st }}$ day } \\
\hline$>15$ & 27 & 90.0 & 25 & 83.3 & \multirow{5}{*}{$0.046 *$} \\
\hline $13-14$ & 0 & 0.0 & 3 & 10.0 & \\
\hline $10-12$ & 2 & 6.7 & 0 & 0.0 & \\
\hline $6-9$ & 1 & 3.3 & 0 & 0.0 & \\
\hline$<6$ & 0 & 0.0 & 2 & 6.7 & \\
\hline \multicolumn{6}{|l|}{$2^{\text {nd }}$ day } \\
\hline$>15$ & 27 & 90.0 & 25 & 83.3 & \multirow{5}{*}{$0.161^{\mathrm{ns}}$} \\
\hline $13-14$ & 0 & 0.0 & 2 & 6.7 & \\
\hline $10-12$ & 2 & 6.7 & 1 & 3.3 & \\
\hline $6-9$ & 1 & 3.3 & 0 & 0.0 & \\
\hline$<6$ & 0 & 0.0 & 2 & 6.7 & \\
\hline \multicolumn{6}{|l|}{$3^{\text {rd day }}$} \\
\hline$>15$ & 27 & 90.0 & 25 & 83.3 & \multirow{5}{*}{$0.449^{\mathrm{ns}}$} \\
\hline $13-14$ & 2 & 6.7 & 2 & 6.7 & \\
\hline $10-12$ & 1 & 3.3 & 1 & 3.3 & \\
\hline $6-9$ & 0 & 0.0 & 0 & 0.0 & \\
\hline$<6$ & 0 & 0.0 & 2 & 6.7 & \\
\hline \multicolumn{6}{|l|}{$4^{\text {th }}$ day } \\
\hline$>15$ & 27 & 90.0 & 27 & 90.0 & \multirow{5}{*}{$0.292^{\mathrm{ns}}$} \\
\hline 13-14 & 1 & 3.3 & 1 & 3.3 & \\
\hline $10-12$ & 1 & 3.3 & 1 & 3.3 & \\
\hline $6-9$ & 0 & 0.0 & 0 & 0.0 & \\
\hline$<6$ & 0 & 0.0 & 0 & 0.0 & \\
\hline
\end{tabular}

* Statistically significant difference $(p<0.05)$ 
Table (7): Percentage distribution of control and study groups regards SOFA score according to kidneys after nursing guidelines implementation.

\begin{tabular}{|c|c|c|c|c|c|}
\hline \multirow{2}{*}{ Creatinine (mg/dl) } & \multicolumn{2}{|c|}{ Control } & \multicolumn{2}{|c|}{ Study } & \multirow[t]{2}{*}{ P.Value } \\
\hline & $\mathrm{No}=(30)$ & $\%$ & $\mathrm{No}=(30)$ & $\%$ & \\
\hline \multicolumn{6}{|l|}{$\mathbf{1}^{\text {st }}$ day } \\
\hline$<1.2$ & 26 & 86.7 & 24 & 80.0 & \multirow{6}{*}{$0.076^{\mathrm{ns}}$} \\
\hline $1.2-1.9$ & 0 & 0.0 & 1 & 3.3 & \\
\hline $2.0-3.4$ & 3 & 10.0 & 0 & 0.0 & \\
\hline $3.5-4.9$ & 0 & 0.0 & 2 & 6.7 & \\
\hline$>5.0$ & 1 & 3.3 & 3 & 10.0 & \\
\hline \multicolumn{5}{|l|}{$2^{\text {nd }}$ day } & \\
\hline$<1.2$ & 26 & 86.7 & 24 & 80.0 & \multirow{5}{*}{$0.251^{\mathrm{ns}}$} \\
\hline $1.2-1.9$ & 1 & 3.3 & 0 & 0.0 & \\
\hline $2.0-3.4$ & 2 & 6.7 & 1 & 3.3 & \\
\hline $3.5-4.9$ & 0 & 0.0 & 2 & 6.7 & \\
\hline$>5.0$ & 1 & 3.3 & 3 & 10.0 & \\
\hline \multicolumn{6}{|l|}{$3^{\text {rd }}$ day } \\
\hline$<1.2$ & 26 & 86.7 & 24 & 80.0 & \multirow{5}{*}{$0.048 *$} \\
\hline $1.2-1.9$ & 3 & 10.0 & 0 & 0.0 & \\
\hline $2.0-3.4$ & 0 & 0.0 & 1 & 3.3 & \\
\hline $3.5-4.9$ & 1 & 3.3 & 1 & 3.3 & \\
\hline$>5.0$ & 0 & 0.0 & 4 & 13.3 & \\
\hline \multicolumn{6}{|l|}{$4^{\text {th }}$ day } \\
\hline$<1.2$ & 26 & 86.7 & 24 & 80.0 & \multirow{5}{*}{$0.019 *$} \\
\hline $1.2-1.9$ & 3 & 10.0 & 0 & 0.0 & \\
\hline $2.0-3.4$ & 1 & 3.3 & 1 & 3.3 & \\
\hline $3.5-4.9$ & 0 & 0.0 & 0 & 0.0 & \\
\hline$>5.0$ & 0 & 0.0 & 5 & 16.7 & \\
\hline
\end{tabular}

$*$ Statistically significant difference $(p<0.05)$

Table (8): Distribution of control and study groups regards patient outcomes.

\begin{tabular}{|c|c|c|c|c|c|}
\hline \multirow{2}{*}{$\begin{array}{l}\text { patient outcomes at the end of ICU } \\
\text { stay. }\end{array}$} & \multicolumn{2}{|c|}{ Control } & \multicolumn{2}{|c|}{ Study } & \multirow{2}{*}{ P. value } \\
\hline & No. $=(30)$ & $\%$ & No. $=(30)$ & $\%$ & \\
\hline ICU stay & \multicolumn{2}{|c|}{$5.4 \pm 1.1$} & \multicolumn{2}{|c|}{$3.6 \pm 0.6$} & $<0.001 * *$ \\
\hline \multicolumn{6}{|c|}{ Complications of emergency hypertension . } \\
\hline CNS comprise & 5 & 16.7 & 3 & 10.0 & \multirow{3}{*}{$0.353^{\mathrm{ns}}$} \\
\hline Cardiovascular comprise & 24 & 80.0 & 19 & 63.3 & \\
\hline Acute renal failure & 6 & 20.0 & 3 & 10.0 & \\
\hline
\end{tabular}

$*$ "Statistically significant difference $(p<0.01)$

-CNS : Central Nervous System

Table (1): Illustrates demographic data and medical history of control and study groups. Regarding to age, it was noticed that the mean age of control and study groups $(58.8 \pm 4.5$ and $58.7 \pm 5.6)$ respectively. Regarding to diagnosis, the highest percentage of patients in control and study groups complained from Pulmonary edema, Renal impairment and Unstable Angina $(20 \%$ and $30 \%, 20 \%$ and $13.3 \%, 20 \%$ and $30 \%$ ) respectively. Regarding to medical history patients had, it was noticed that there were no statistically significant difference between control and study groups.

Figure(1): This figure reveals that, the majority of patients were males in control and study groups $(66.7 \%$ and $80.0 \%)$ respectively.

Figure(2): This figure shows that, the majority of patients in control and study groups were married $(76.7 \%$ and $66.7 \%)$ respectively. 
Table (2) Illustrates respiratory and cardiovascular assessment of control and study groups. As regards to On admission blood pressure measurements, it was found that there were Statistically significant difference between control and study groups in Right arm SBP, standing SBP, Right arm DBP, Left arm $\mathrm{DBP}$ and standing $\mathrm{DBP}(\mathrm{P}=0.009, \mathrm{P}=0.024, \mathrm{P}=0.028$, $\mathrm{P}=0.012$ and $\mathrm{p}=0.028$ ) respectively. As regards to Heart rate, Respiratory rate, dysrhythmia, Cyanosis and Chest assessment of studied groups our study mentioned that there is no statistically significant difference

Table (3): Shows surgical and family history of control and study groups. Regards to surgical history patients had, the majority of patients in control and study groups had no surgery $(73.3 \%$ and $83.3 \%)$ respectively. Regards to family history of hypertension it was noticed that there were statistically significant difference between control and study groups $(\mathrm{P}=0.004)$.

Table (4): Shows distribution of control and study groups as regards temperature, heart rate and respiratory rate assessment. This study mentioned that there is no statistically significant difference between control and study groups.

Table (5): Shows distribution of control and study groups as regards blood pressure assessment. Regarding to base line measurement of blood pressure on admission, results revealed that there were statistically significant difference between control and study groups in SBP $(\mathrm{P}=0.009)$ and $\mathrm{DBP}(\mathrm{P}=0.028)$.Regards to measurement of blood pressure after 45 minutes of hospitalization, results revealed that there were statistically significant difference between control and study groups in $\operatorname{SBP}(\mathrm{P}=0,001)$ and $\mathrm{DBP}(\mathrm{P}=0.005)$. $)$. Regards to measurement of blood pressure after 60 minutes of hospitalization, results revealed that there were statistically significant difference between control and study groups in $\operatorname{SBP}(\mathrm{P}=0.007)$ and $\mathrm{DBP}$ $(\mathrm{P}=0.003)$. Regards to measurement of blood pressure during first day of hospitalization, results revealed that there were statistically significant difference between control and study groups in $\operatorname{SBP}(\mathrm{P}=0.001)$ and DBP $(\mathrm{P}=0.007)$

Table (6): shows distribution of control and study groups as regards SOFA score according to nervous system. As regards to Glasgow coma scale in 1st day, results revealed that there were statistically significant difference between control and study groups $(\mathrm{P}=0.046)$.

Table (7): shows distribution of control and study groups as regards SOFA score according to kidney function. As regards to Creatinine in $3^{\text {rd }}$ day and $4^{\text {th }}$ day results revealed that there were statistically significant difference between control and study groups $(\mathrm{P}=0.048$ and $\mathrm{P}=0.019)$ respectively.

Table (8): Show distribution of control and study groups as regards patient outcomes. As regards to ICU stay, control group stay in hospital days with average of 5.4 while study group average were 3.6 days and there is statistically significant difference between the studied groups $(\mathrm{p}<0.001)$. Regarding to complications of emergency hypertension patients had on admission, the majority of patients in control and study groups had Cardiovascular comprise symptoms $(80 \%$ and $63.3 \%)$ respectively, followed by acute renal failure (20\%and $10 \%)$ respectively and CNS comprise (16.7\%and10\%) respectively in control and study

\section{Discussion}

Hypertensive emergency nursing guideline is paramount importance for coronary intensive care unit patients to avoid hypertensive emergency complications that have a devastating effect on the quality of life in those who suffer from hypertensive emergency. The focus of nursing management for the patient with hypertensive emergency is to return the blood pressure to the desired range without introducing other complications as a result of therapy. After the hypertension is controlled, the nurse identifies the factors that resulted in this lifethreatening condition. Several nursing diagnoses are associated with hypertensive emergency (Lewis et al., 2016).

A variety of hypertensive emergency care regimens are available for critical patients, but nothing is conclusive so far. The basic principle for preventing hypertensive emergency complication is meticulous and protocolized care. The aim and objective of intensive nursing care is to provide general and specific critical care plan to each patient (Cline $\&$ Amin 2013)

The present study clarified that there was no statistically significant difference between experimental and control groups with respect to age. This result was in line with Marques et al., (2016) who reported that there was no statistically significant difference between experimental and control groups with respect to age.

As regard the sex, the current study revealed that the majority of patients were males in control and study groups. Also, this finding is nearly similar to the results of Nayak, et al., (2014) who found that majority of patients with hypertensive emergency were males. This disagree with Bennett \& Shea (2008) who reported that his study results indicated that female were significantly over-represented compared to males . 
As the researcher point of view, the majority of patients were males in control and study group. As males have more stressors than females. Males are responsible for their work and their families' financial affaires. They usually think about their kid's future and are engaged with political affaires. Many of males didn't used to express feeling with others.

In the current study, concerning their marital status about two thirds of the patients were married in control and study groups. This finding is nearly similar to the result of Marques et al., (2016) who found that two thirds of studied sample were married. As the researcher point of view, the two thirds of the patients were married in the studied groups. As married patients have more stressors than single, divorced or widow patients. Married people are responsible for supplying their kids with good care, teaching and they usually anxious about their kids' future. Married people are always engaged with financial affaires and how to use it to satisfy family needs. Many of married people can't understand each other and have many conflicts and problems because of boring family life routine.

As regards to diagnosis, the forth of patients with acute pulmonary edema in the studied groups. Also, this finding is nearly similar to the results of Pinna, et al., ( 2014) that found that forth of hypertensive emergency patients had acute pulmonary edema .

Regarding to other medical problem in addition to hypertension patients had, it was noticed there were statistically significant difference between control and study groups who suffer from bronchial asthma and angina chest pain. This finding is nearly similar to the results Marques, et al., (2016) who reported that the commonest presenting complaints were neurological deficits followed by dyspnea and chest pain and patient presented with convulsions and visual deficits. Also, this agree with Vilela-Martin et al., (2011) who noticed were the most common headache and chest pain, the most frequent were dyspnea, and neurological deficit who reported that the percent of patients with emergency hypertension acute pulmonary edema and the percent of patients with emergency hypertension acute myocardial Infarction. Also, this finding is nearly similar to the results of Pinna, et al., (2014) that found of hypertensive emergency patients had acute pulmonary edema.

The current study revealed statistically significant difference between control and study groups in systolic and diastolic blood pressure for right and left arm. This is in same line with Clark et al., (2012) who documented that differences in systolic blood pressure between arms can predict an increased risk of cardiovascular events and all causes mortality over 10 years in people with hypertension. This difference could be a valuable indicator of increased cardiovascular risk.

Regarding to family history of hypertension it was noticed that there was statistically significant difference between control and study groups. This agree with Nayak, et al.,( 2014) who reported that family history, ethnicity, waist circumference and hypertension are more significant risk factors of this disease than BMI and gender in the Trinidadian population.

As the researcher point of view, family history of hypertension it was noticed that there was statistically significant difference between control and study groups, people with family history of hypertension didn't receive enough about lines of primary disease prevention from community health nurse as life style modifications and early detection of disease by frequent monitoring.

In the current study, known hypertensive patients in control and study groups were at higher risk of presenting with acute target organ damage associated with the hypertensive emergency. This in line with Vilela-Martin et al., ( 2011) who found that known hypertensive were at a higher risk of presenting with acute target organ damage associated with hypertensive emergency.

As regarding nervous system the Glasgow coma scale in first day revealed there was statistically significant difference between control and study group. This is nearly similar with Pinna et al.,( 2014) who reported that among 391 patients with hypertensive emergencies, forth patients had acute pulmonary edema, followed by patients had stroke, myocardial infarction, acute aortic dissection, acute renal failure, then hypertensive encephalopathy.

The present study revealed that creatinine in third and fourth days there were statistically significant difference between control and study groups. This is agree with Ruilope et al., (2012) who documented serum creatinine is a very powerful predictor of cardiovascular events and death. Reduced renal function does not preclude that the diastolic BP target is achieved. There is, however, a small group of patients whose renal function deteriorates despite satisfactory reduction of diastolic BP.

As regards to ICU stay, the current study revealed that control group stay in hospital was longer than hospital stay of study group and there was statistically significant difference between control and study groups. This is in line with Benjamin, et al., ( 2013) who documented periods of high hospital stay with hypertension in emergency department crowding were associated with increased in patient mortality and modest increases in length of stay and costs for admitted patients. 
The current study showed that majority of patients had cardiovascular comprise symptoms followed by Acute renal failure and CNS comprise in control and study groups. This finding disagrees with Cline \& Amin (2013) who found that the commonest presenting complaints were neurological comprise followed by dyspnea and chest pain.

Finally, in the fact that study documented that the emergency hypertension nursing care guidelines play a vital role in improvement of patient's outcomes with emergency hypertension. Emergency hypertension patients must be assessed and monitored closely for any deterioration in condition and the relevant emergency hypertension care plan or pathway must be implemented.

\section{Conclusion}

Based on the results of this study, it could be concluded that: implementing of emergency hypertension nursing care guideline including assessment of patient blood pressure using standardized blood pressure measuring technique, assessment of degree of organ system dysfunction using SOFA score, assessment of laboratory investigations obtained, assessment of cardiovascular system, renal system and neuromuscular system. Then applying nursing care specific to the affected organ that are significantly effective in reduction incidence of complications occurrence in critically ill patients with hypertensive emergency.

Based on the study finding, the following recommendations are suggested:-

\section{Recommendations}

- Emergency hypertension nursing care guidelines should be standardized as a basic part of care provided to all emergency hypertensive patients in coronary care units.

- Equip intensive care units with simple illustrated booklet about emergency hypertension nursing care guideline.

- Improve documentation of hypertensive emergency complications in a record, to measure the size of this problem accurately

- Provide the patient with a pamphlet illustrates emergency hypertension as acute phase and the importance of continuous and regular oral taking of antihypertensive medications as prescribed.

- Provide patient education for critical ill patients regarding emergency hypertension.

- Reapply this research on a larger probability sample acquired from different geographical areas in Egypt for generalization.

\section{References}

1. Nayak, B., Sobrian, A., Latiff, K., Pope, D., Rampersad, A., Lourenço, K., \& Samuel, N., (2014): The association of age, gender, ethnicity, family history, obesity and hypertension with type 2 diabetes mellitus in Trinidad. Diabetes \& Metabolic Syndrome: Clinical Research \& Reviews, 8(2), 91-95..

2. Benjamin C., (2013): Effect of Emergency Department Crowding on Outcomes of Admitted Patients. Annual of emergency medicine an international journal, 61(6), pp.1-7.

3. Bennett, N., \& Shea, S., (2008): Hypertensive emergency: Case criteria, sociodemographic profile, and previous care of 100 cases. American Journal of Public Health, 78(6), pp.636-640.

4. Carlson, K., (2011): AACN advanced critical care nursing. St. Louis, MO: Saunders/Elsevier. Pp 501-520

5. Clark, C., Taylor, R., Shore, A., \& Campbell, J., (2012): The difference in blood pressure readings between arms and survival: primary care cohort study. Bmj, 344, e1327.Available at: http://www.pubmedcentral.nih.gov/

6. Cline, D., \& Amin, A., (2013): Drug Treatment for Hypertensive Emergencies Objectives : International Emergency Medicine and Cardiac Research Education Group, 1(January), pp.1-12..

7. Lewis, S., Bucher, L., Heitkemper, M., Harding, M., Kwong, J., \& Roberts, D., (2016): Medical-Surgical Nursing: Assessment and Management of Clinical Problems 10th ed., Canada: Elsevier. Available at: www.elsevier.com.

8. Mancia, G., \& Fagard, R., (2014): Hypertensive urgencies and emergencies. Journal of Hypertension, 32(9), 1911.

9. Marques, P., Nogueira-Silva, L., Lima, M., Cunha, F., \& Pereira, C., (2016). determinants of osa in patients of a hypertension clinic in a hypertensive population in the south. Wolters Kluwer Health, Inc. All rights reserved, 34(September), p.2016.

10. Mohan, A., \& Nelson-Piercy, C., (2016): Hypertensive Patient. Gynecologic and Obstetric Surgery Challenges and Management Options, 22-24.

11. Pinna, G., Pascale, C., Fornengo, P., Arras, S., Piras, C., Panzarasa, P., \& Pietrelli, S., (2014): Hospital admissions for hypertensive crisis in the emergency departments: a large multicenter Italian study. PLoS One, 9(4), 
12. Ruilope, L., salvetti, A., jamerson, K., hansson, L., warnold, i., wedel, H., \& zanchetti, A., (2012): Renal function and intensive lowering of blood pressure in hypertensive participants of the hypertension optimal treatment (HOT) study. Journal of the American Society of Nephrology: JASN, 12(2), pp.218-225.

13. Sinha, A., (2011): Chapter-14 Hypertensive Emergencies. Principles of Pediatric and Neonatal Emergencies, 152-157 .

14. Vilela-Martin, J., Vaz-de-Melo, R., Kuniyoshi, C., Abdo, A., \& Yugar-Toledo, J., (2011): Hypertensive crisis: clinical-epidemiological profile. Hypertension Research, 34(3), 367-371.

15. Gupta, P., Gupta, H., \& Khoynezhad, A., (2009): Hypertensive emergency in aortic dissection and thoracic aortic aneurysm-a review of management. Pharmaceuticals, 2(3), 66-76.

16. Stevens, K., (2013): The impact of evidencebased practice in nursing and the next big ideas. OJIN: The Online Journal of Issues in Nursing, 18(2), 1-13.

17. Hasan, D., Emeash, A., Mustafa, S., Abdelazim, A., \& Alaa El-din, A., (2014): Hypertension in Egypt: A Systematic Review. Current hypertension reviews, 10(3), 134-141.

18. World Health Organization. (2013): A global brief on hypertension: silent killer, global public health crisis: World Health Day 2013.

19. Vincent, J., De Mendonça, A., Cantraine, F., Moreno, R., Takala, J., Suter, P., \& Blecher, S., (2015): Use of the SOFA score to assess the incidence of organ dysfunction/failure in intensive care units: results of a multicenter, prospective study. Critical care medicine, 26(11), 1793-1800. 
